\title{
MÉTOdOS Y METOdOLOGÍAS UTILIZADOS EN EL PROCESO DE ENSEÑANZA-APRENDIZAJE
}

\author{
AUTORES: Washington Narváez Campana ${ }^{1}$ \\ Cruz Victoria Ponce Zavala² \\ Raquel Vera Velázquez ${ }^{3}$ \\ Kirenia Maldonado Zúñiga ${ }^{4}$
}

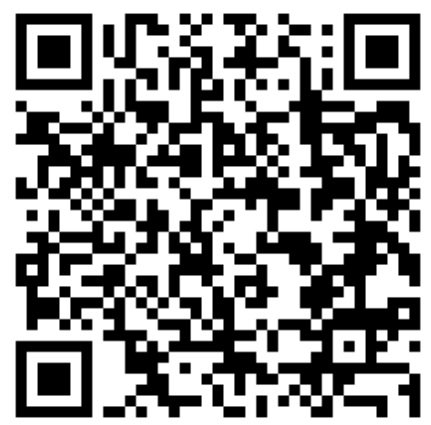

DIRECCIÓN PARA CORRESPONDENCIA: washington.narvaez@unesum.edu.ec

Fecha de recepción: 25/01/2020

Fecha de aceptación: 24/02/2020

\section{RESUMEN}

Los métodos de enseñanza son los componentes más dinámicos del proceso de enseñanzaaprendizaje, pues están basados en las acciones de los profesores y estudiantes. Algunos autores separan los métodos de enseñanza de los métodos de aprendizaje, cuando en realidad están íntimamente relacionados y forman una unidad dialéctica. Al aplicar un método de enseñanza hay que tener presente las operaciones lógicas que predominan en cada etapa del proceso de aprendizaje y priorizar las que facilitan la actividad independiente y creadora de los estudiantes. Estos métodos son muy variados, pues existen diversos criterios de clasificación. El objetivo de este trabajo es relacionar los métodos y metodologías de enseñanza- aprendizaje teniendo en cuenta los criterios de diferentes autores con la definición original y explicar brevemente los aspectos fundamentales de los métodos y metodologías, destacando su concepto y clasificación, así como su aplicación en las distintas etapas del aprendizaje. Entre las clasificaciones más difundidas se describen de forma resumida las correspondientes al grado de participación de los sujetos, el nivel de asimilación de los contenidos de enseñanza y los métodos problémicos. Por último, se explican las etapas de proceso de aprendizaje y se precisan los métodos de enseñanza que se aplican en cada una de ellas y persigue los siguientes objetivos: proporcionar a los docentes una herramienta para el diseño de

\footnotetext{
${ }^{1}$ Docente investigador de la Asignatura de Bioética y Problemas Actuales de la Ciencia, Carrera de Ingeniería Agropecuaria, Facultad de Ciencias Naturales, Universidad Estatal del Sur de Manabí, Campus Los Ángeles, vía Noboa Km 1 1 1/2 s/n, en Jipijapa, Manab

${ }^{2}$ M.Dc. Docente investigador de la Asignatura de Expresión Oral y Escrita, Carrera de Ingeniería Agropecuaria, Facultad de Ciencias Naturales, Universidad Estatal del Sur de Manabí, Campus Los Ángeles, vía Noboa Km 1 1⁄2 s/n, en Jipijapa, Manabí

${ }^{3}$ M.Sc. Docente investigador de la Asignatura de Matemáticas y Algebra, Carrera de Ingeniería Agropecuaria, Facultad de Ciencias Naturales, Universidad Estatal del Sur de Manabí, Campus Los Ángeles, vía Noboa Km 1 1⁄2 s/n, en Jipijapa, Manabí.

${ }^{4}$ M.Sc. Universidad Estatal del Sur de Manabí, Facultad de Sistemas Computacionales, Jipijapa, Ecuador.
} 
Washington Narváez Campana, Cruz Victoria Ponce Zavala, Raquel Vera Velázquez...

guías académicas, ayudar a los procesos de diseño de acciones formativas para el profesorado en el desarrollo del proceso de enseñanza- aprendizaje y aportar a la investigación en este campo.

PALABRAS CLAVE: Competencia, meta, método de enseñanza, metodología docente, aprendizaje.

\section{METHODS AND METHODOLOGIES USED IN THE TEACHING-LEARNING PROCESS}

\section{ABSTRACT}

Teaching methods are the most dynamic components of the teaching-learning process, since they are based on the actions of teachers and students. Some authors separate teaching methods from learning methods, when in reality they are closely related and form a dialectical unit. When applying a teaching method, it is necessary to keep in mind the logical operations that predominate at each stage of the learning process and prioritize those that facilitate the independent and creative activity of the students. These methods are very varied, since there are different classification criteria. The objective of this work is to relate the teaching-learning methods and methodologies taking into account the criteria of different authors with the original definition and to briefly explain the fundamental aspects of the methods and methodologies, highlighting their concept and classification, as well as their application in the different stages of learning. Among the most widespread classifications, those corresponding to the degree of participation of the subjects, the level of assimilation of the teaching content and the problem methods are briefly described. Finally, the stages of the learning process are explained and the teaching methods that are applied in each one are specified, and the following objectives are pursued: to provide teachers with a tool for the design of academic guides, to help the learning processes design of training actions for teachers in the development of the teaching-learning process and contribute to research in this field.

KEYWORDS: Competence, goal, teaching method, teaching methodology, learning.

\section{INTRODUCCIÓN}

Los métodos de enseñanza son los componentes más dinámicos del proceso de enseñanzaaprendizaje, pues están basados en las acciones que realizan los profesores y estudiantes, las que a su vez comprende una serie de operaciones dirigida a lograr los objetivos propuestos en este proceso. (Salas Perea, RS, 1999)

Según (Ruiz Socarrás, José Manuel,1994) método de enseñanza es el medio que utiliza la didáctica para la orientación del proceso enseñanza-aprendizaje. Etimológicamente el termino método proviene del griego mhtodos que significa camino, vía, medio para llegar al fin. En la ciencia el método se define como un sistema de reglas que nos sirven para alcanzar un objetivo determinado y que persigue también los mejores resultados.

Algunos pedagogos separan los métodos de enseñanza de los métodos de aprendizaje, o sea, que consideran la enseñanza y el aprendizaje por separado. En realidad, estos dos aspectos están íntimamente relacionados y no se deben separar, pues la esencia del proceso de enseñanza reside en la interacción entre el profesor y el estudiante, que forman una unidad dialéctica. 
Desde el punto de vista etimológico la palabra método significa "camino hacia una meta" y en un sentido más amplio es el modo razonado de obrar, manera de ordenar la actividad para alcanzar un objetivo. En general, todo método se puede definir como un sistema de acciones sucesivas y conscientes del hombre, que tiende a alcanzar un resultado, que se corresponde con el objetivo trazado.

Por todo lo antes expuesto se puede considerar como método de enseñanza los modos de actuación de los profesores y alumnos, que se realizan de forma ordenada e interrelacionada, con el objetivo de facilitar a los educandos la asimilación del contenido de enseñanza. Indican cómo enseñar. (Álvarez de Zayas C, 1999)

La clasificación de los métodos de enseñanza es un problema aún no resuelto en la Ciencia Pedagógica, pues existen diversos criterios al respecto y no hay uno que sea aceptado por todos. Por lo tanto, no existe un método de enseñanza universal y muchas son las posibilidades de combinarlo, en dependencia de diversos factores, como las particularidades de los estudiantes y la etapa del proceso de aprendizaje que se desarrolla, los objetivos y contenidos de enseñanza, los medios disponibles, la forma organizativa de la actividad docente y, por supuesto, el trabajo creador del profesor.

Entre las diversas clasificaciones existentes acerca de los métodos de enseñanza, basadas en el criterio de diferentes autores, se podrían citar como ejemplos, las siguientes: (Martínez Yantada, M, 1987)

- Según las vías lógicas de obtención de los conocimientos (inductivos, deductivos y analíticosintéticos).

- Según las fuentes de obtención de los conocimientos o por la forma de percepción (orales, visuales y prácticos).

- Según la combinación de métodos de enseñanza y aprendizaje o binarios.

En la actualidad, las clasificaciones más difundidas de los métodos de enseñanza, son las siguientes:(Álvarez de Zayas C,1999)

-Según el grado de participación de los sujetos o de interrelación profesor- estudiante (expositivo, trabajo independiente y elaboración conjunta).

-Según el grado de dominio o nivel de asimilación del contenido de enseñanza (pasivos o reproductivos y activos o productivos, incluyendo en estos últimos los métodos problémicos).

Los autores (Labarrere, G y Valdivia P, G.E, 1991); (Silvestre, 2002); Caballero, 2012) unen unos métodos con otros como muestra de su similitud (analítico-sintéticos, explicativo-ilustrativo), pero estos parecidos no son compartidos por (García Batista, G, 2002); (Addine, 2004), quienes consideran cada método por separado. 
Washington Narváez Campana, Cruz Victoria Ponce Zavala, Raquel Vera Velázquez...

Según (Ortega, Loubon y Franco, César, 2010) consideran las formas académicas de organizar el proceso como métodos de enseñanza, dígase clase magistral, taller y expresan las razones de la diversidad de estas posiciones, de la dicotomía método de enseñanza-aprendizaje, y de la inclusión de las formas académicas de organizar el proceso como métodos de enseñanza estriban en algunas imprecisiones que se presentan en las definiciones universalmente aceptadas del concepto método de enseñanza.

(Alcoba González, J, 2012) expresó: un método de enseñanza es el conjunto de técnicas y actividades que un profesor utiliza con el fin de lograr uno o varios objetivos educativos, que tiene sentido como un todo y que responde a una denominación conocida y compartida por la comunidad científica. (p. 96). Las condiciones implícitas en esta definición también pueden generar desconcierto, pues hay otros componentes del proceso en los que se articula un conjunto de actividades con el fin de lograr los objetivos educativos. Aunque hay que resaltar que en esta definición no se infiere que el método de enseñanza devenga en método de aprendizaje.

Los métodos dependen, de los objetivos y del contenido de la educación en un momento determinado, según el nivel de enseñanza de que se trate, lo que condiciona cuándo resulta más oportuno utilizar uno u otro. A su vez constituyen un instrumento de ayuda al educador, para ejercer influencia en la conciencia, los sentimientos y las actitudes y conductas manifiestas de los alumnos. La utilización adecuada de estos, tanto en su sistematicidad como en su coherencia, contribuye de una manera directa al logro de la educación y formación de la personalidad, en correspondencia con el fin y los objetivos propuestos en una sociedad determinada.

Resulta de interés también clarificar el significado del término metodología. Según el Diccionario de la Real Academia, la metodología es la "ciencia del método", o bien el "conjunto de métodos que se siguen en una investigación científica o en una exposición doctrinal". (RAE, 2008). En la primera acepción nos estamos refiriendo por tanto a un conocimiento científico y en la segunda a un conjunto de métodos. Desde este punto de vista, metodología educativa es, en primer lugar, el área de la Ciencia que estudia los métodos de enseñanza. Y, en segundo lugar, la metodología es el conjunto de métodos utilizados por un docente.

La metodología es uno de los elementos imprescindibles en la elaboración de unidades didácticas desde que fuera incluida en el modelo de (Fernández, A y Sarramona, J, 1975). Sin embargo, y pese a lo mucho que se ha escrito sobre este tema, aún es posible encontrar dificultades en la utilización y clasificación de los métodos y metodologías de enseñanza en la clase del nivel superior. Se trata de un problema importante, pues por un lado el profesorado tiene falta de metodologías para desarrollar el proceso de enseñanza-aprendizaje y por otro lado la formación del estudiantado como ente fundamental en este proceso.

Es un elemento esencial del proceso de formación, porque constituye la manera, la forma cómo se lleva a cabo la formación. La finalidad principal del escogimiento de una metodología adecuada, es que los estudiantes, aprendan.

Desde el punto de vista del constructivismo, se considera que la metodología debe reunir varias características, que ya son mencionadas en otras fuentes y de las cuales se hace un resumen a continuación (Universidad San Buenaventura, 2015): tomar en cuenta el contexto: los conocimientos deben ser globales y particulares, a la vez. Esto requiere un equilibrio entre la revisión teórica de los contenidos, pero también su aplicación particular en los contextos específicos en los cuales los estudiantes tienen que desenvolverse. 
Es importante plantear que una metodología didáctica supone una manera concreta de enseñar, método supone un camino y una herramienta concreta que utilizamos para transmitir los contenidos, procedimientos y principios al estudiantado y que se cumplan los objetivos de aprendizaje propuestos por el profesor. Pero, ¿qué hay detrás de la elección de una metodología didáctica? (Hernández, P, 1997): es un elemento esencial del proceso de formación, porque constituye la manera, la forma cómo se lleva a cabo la formación. La finalidad principal del escogimiento de una metodología adecuada, es que los estudiantes, aprendan.

Una de las primeras dificultades que surge tras el análisis de distintos autores es la gran dispersión que existe en la enumeración de métodos y metodologías. Así, por ejemplo, la relación del (Ministerio de Educación y Ciencia MEC, 2006) incluye solo tres métodos, pero la de (Navaridas Nalda, F, 2004) incorpora veinticinco y en esta investigación relacionamos treinta y ocho incorporando otros criterios de autores según tabla \# 3.

Otra dificultad es que los autores no utilizan los mismos términos para referirse a métodos que parecen similares. Así por ejemplo (Miguel, M y Amat, O, 2002); (Miguel, M, 2006) usan el término lección magistral, mientras que la Universidad Rovira Virgili se refiere a este método como sesión magistral (URV, 2006) y (Zabalza, M.A, 2003) citando a (Tejada Jiménez, H. y Hernández Jorge, C, 2001) utiliza método magistral, (Jares, X, 2002) cita un método conocido como dinámica de clarificación de valores, pero para el (García Batista, G, 2002) este método se llama ejercicio de clarificación de valores. Otro ejemplo que encontramos donde hablan de estudio de casos en (Miguel, M, 2006); (Navaridas Nalda, F, 2004), mientras que (López Camp, J ,2005) se refiere a ello como método.

Otra dificultad que encontramos es que algunos autores unen unos métodos a otros como muestra de su similitud, pero estos parecidos no son compartidos por otros autores. Así, por ejemplo, (Jares, X, 2002) habla de juegos y ejercicios de simulación y (Fernández March, 2006) de simulación y juego.

Sin embargo, (Navaridas Nalda, F, 2004) y el (TEC de Monterrey, 2006) solo hablan de simulación (Navaridas Nalda, F, 2004) por otro lado, (Portela Leiva, G; Morales Suárez, I, 2005) escribe simulación/role playing, pero el juego de roles aparece aislado en otros autores, como por ejemplo en (Amat, O, 2002 y López Camps, J, 2005). Para este último autor, finalmente, el método del caso forma parte de los juegos y simulaciones, mientras que en otros autores encontramos ambos métodos por separado, como por ejemplo en (Navaridas Nalda, F, 2004) ; ( Addine Fernández, F, 2004); (Fernández March, 2006) y (Alcoba González, J, 2012)

Por último, si nos fijamos en los objetivos que pretenden los diversos métodos, tampoco parece haber acuerdo entre los distintos autores. Por ejemplo, Amat refiere que los métodos que fomentan la creatividad son la tutoría, el Phillips66 y la tormenta de ideas (Amat, O,2002) y sin embargo para Navaridas la tutoría es uno de los métodos orientados al cambio de conocimientos, concretamente una de las estrategias individualizadoras. Para él, los métodos que fomentan la creatividad son el torbellino de ideas, la interrogación didáctica, las técnicas audiovisuales y el método del incidente (Navaridas Nalda, F, 2004), Bourner, sin embargo, amplía el listado de métodos que sirven para que el estudiante genere ideas a diez, entre los que resulta llamativo encontrar las técnicas de relajación (Bourner, T, 1997)

En fin, las definiciones hasta ahora universalmente estandarizadas sobre el método de enseñanza generan confusión entre los estudiosos de la temática. Como bien se ha ilustrado con los ejemplos 
Washington Narváez Campana, Cruz Victoria Ponce Zavala, Raquel Vera Velázquez...

analizados anteriormente, no han permitido dilucidar con exactitud las condiciones necesarias y suficientes para diferenciar el método de enseñanza del método de aprendizaje, y de otros componentes como los medios de enseñanza y las formas académicas de organización con los que algunos autores confunden.

De acuerdo con (Álvarez de Zayas, 1999) (...) la forma está dialécticamente relacionada con el método, mientras la forma atiende la organización externa del proceso, el método atiende la organización interna. El método es la esencia de la forma, esta (la forma) el fenómeno de aquel (del método); ambos son componentes operacionales del proceso que expresan su dinámica (...). (p. 23)

La idea anterior permite esclarecer la brecha epistemológica que aparece en las definiciones sobre método de enseñanza, y determinar las condiciones necesarias y suficientes que conforman las definiciones de método de enseñanza y método de aprendizaje.

Por un lado, el método de enseñanza es la vía o camino para llegar al objetivo, por otro lado, el método expresa la secuencia de acciones, actividades y operaciones del profesor para trasmitir un contenido de enseñanza. También, el método de enseñanza atiende la organización interna del proceso de enseñanza, por lo que se infiere que esta organización interna se expresa dentro de determinada forma académica de organización, que a su vez atiende la organización externa del proceso.

El método no es en ninguna medida la forma académica de organización del proceso de enseñanza, es la esencia de esta, como bien expresara en (Álvarez de Zayas, 1999). La naturaleza de la forma académica de organización se expresa a través de los métodos de enseñanza que constituyen la secuencia de acciones, operaciones o actividades que la distinguen de otros componentes operacionales del proceso de enseñanza.

Por tanto, según las consideraciones de los autores del presente artículo, el método de enseñanza es la secuencia de acciones, actividades u operaciones del que enseña que expresan la naturaleza de las formas académicas de organización del proceso para el logro de los objetivos de enseñanza.

Apoyándose en esta definición, las clasificaciones de los métodos de enseñanza se realizarán teniendo en cuenta diferentes criterios científicos y se evitarán confusiones.

A partir de estas consideraciones es posible también redefinir el concepto de método de aprendizaje como la secuencia de acciones, actividades u operaciones del que aprende para la adquisición y asimilación del contenido de enseñanza con los consiguientes cambios en su sistema de conocimientos y en su conducta.

Al igual que el método de enseñanza, el método de aprendizaje atiende la estructura interna de la forma académica de organización, pero se expresa dentro y fuera de esta. Pues, según (Ortega Loubon, C y Franco, J.C, 2010) “(...) el aprendizaje es el proceso por el cual los organismos modifican su conducta para adaptarse a las condiciones cambiantes del medio que los rodea. Es el modo principal de adaptación de los seres vivos (...)”. (p. 1). Por tanto, el aprendizaje escolar puede considerarse como un cambio en el sistema nervioso que resulta de la experiencia del alumno 
dentro y fuera del contexto educativo, y que origina cambios duraderos en su conducta y en su sistema de conocimientos.

De modo que, en un escenario educativo, el que enseña puede utilizar métodos orales, pero el que aprende utiliza otros métodos para adicionar, seleccionar, integrar, o retener y fijar el contenido que se le trata de trasmitir. Así el método de enseñanza tiene una intención, y el método de aprendizaje tiene otra; el método de enseñanza se proyecta hacia un resultado, y el de aprendizaje se proyecta hacia otros. A través del método de enseñanza el que enseña trasmite información, a través del método de aprendizaje el que aprende procesa e integra esa información o parte de ella que le resulta útil o significativa.

En el método de enseñanza se expresan procesos de exteriorización; y en el de aprendizaje procesos de interiorización asociados a la aparición de nuevas sinopsis o reorganización de las existentes, se añaden conexiones, se modifican otras o se eliminan las inútiles, se prueban combinaciones y seleccionan las mejores, se generan procesos de adición y sustracción de contenidos.

De manera que, en la medida en que un profesor utiliza un sistema de métodos de enseñanza para trasmitir un contenido, el estudiante utiliza otro sistema de métodos de aprendizaje para interiorizar lo que le resulta útil y significativo de este contenido de enseñanza.

Por ejemplo, un profesor expone un contenido de enseñanza, lo cual implica que se apoya en otros métodos como la explicación, ilustración, demostración, argumentación, fundamentación, narración, descripción; y en la misma medida en que transcurre el accionar del profesor, el estudiante emplea otros métodos que le permiten interiorizar aquellos contenidos que le impresionan, les son útiles y significativos. En ese caso, se puede apoyar en la toma de notas, la elaboración de esquemas, mapas conceptuales, modelos, resúmenes, entre otros.

En fin, cuando el profesor expone, explica, ilustra, demuestra, argumenta, fundamenta; el estudiante resume, modela, adiciona, selecciona, asocia, integra, elimina, combina.

Tal y como hemos escrito líneas arriba, la investigación en métodos de enseñanza conlleva una serie de dificultades que hemos pretendido superar a través de las acciones que pasamos a enumerar.

Como aproximación a estos problemas el presente trabajo propone una delimitación conceptual y muestra el proceso de construcción de un listado de métodos y metodologías de enseñanzaaprendizaje utilizado en una investigación sobre la estrategia docente de las instituciones de Educación Superior (Alcoba González, J ,2012) así como la versión actualizada de dicha relación en la tabla \# 2.

Es común en la literatura científica sobre métodos de enseñanza encontrar listados de métodos que cada autor enumera según su experiencia y conocimiento, siendo ciertamente frecuente que unos autores se apoyen en la obra de otros. Esto posiblemente es la causa de los diversos problemas que surgen en la investigación en este campo, como son los que mencionamos a continuación.

El objetivo de este trabajo es explicar brevemente los aspectos fundamentales de los métodos y metodologías de enseñanza-aprendizaje, destacando su concepto y clasificaciones más usuales, así como su aplicación en cada etapa del proceso de aprendizaje, por lo que después de consultar varios 
Washington Narváez Campana, Cruz Victoria Ponce Zavala, Raquel Vera Velázquez...

autores relacionamos 38 métodos y metodologías de las más utilizadas y estudiadas por los diferentes autores analizados.

Por todo los antes planteado y por dificultades reflejadas en la heteroevaluación realizada por los estudiantes a los docentes en cada período académico de la Universidad Estatal de Manabí aplicamos una encuesta sobre el dominio y utilización de los métodos de enseñanza-aprendizaje utilizados en el nivel superior, lo hicimos en las carreras de Agropecuaria y Computación y Redes con el fin de proponer algunas estrategias para resolver las dificultades existentes.

\section{DESARROLLO}

\section{MATERIALES Y MÉTODOS}

La presente investigación se realizó en el año 2019 en la Carreras de Agropecuaria y Computación y Redes de la Universidad Estatal del Sur de Manabí, ubicada en el Campus Los Ángeles, vía Noboa Km 1 1 1/2 s/n, en Jipijapa, Manabí

Se elaboró una encuesta partiendo de los planteamientos de los diferentes autores consultados y considerando los criterios orientadores sobre el dominio y aplicación de los métodos de enseñanzaaprendizaje derivados del marco teórico, de ocho preguntas cerradas y para su construcción se analizaron escalas previamente validadas en diferentes investigaciones, analizando los temas sobre el proceso de enseñanza- aprendizaje:

1-Funciones didácticas de la clase.

2-Los métodos de enseñanza -aprendizaje.

3-Las metodologías de aprendizaje.

Se aplicó la encuesta a docentes de las carreras antes mencionadas sobre los métodos de enseñanzaaprendizaje aplicados en clases teniendo en cuenta las funciones didácticas de la clase, los métodos y metodologías utilizadas en el desarrollo del proceso de enseñanza- aprendizaje y los contenidos de las asignaturas en las carreras de agropecuaria y computación y redes de la Universidad Estatal del Sur de Manabí como se muestra en la siguiente tabla \# 1. Gráfico \# 1. Tamaño de la muestra.

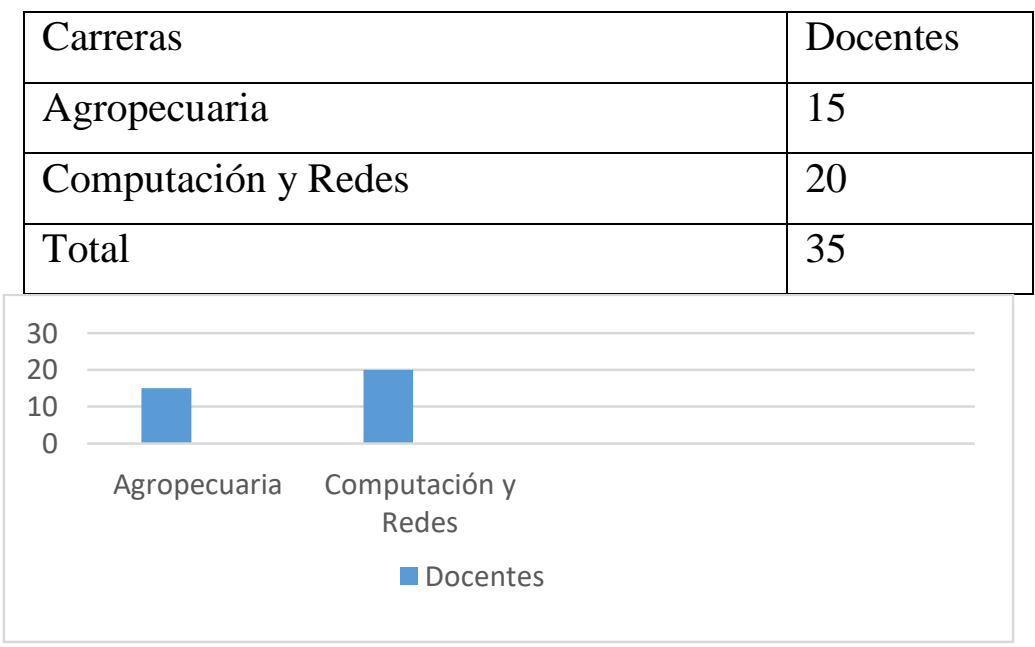

Todo esto nos lleva a considerar que el profesor podrá contribuir al desarrollo de estrategias de aprendizaje en sus estudiantes siempre y cuando tenga mayores conocimientos de los métodos y metodologías de enseñanza- aprendizaje. 
El instrumento aplicado estuvo dirigido a medir el nivel de conocimiento que tienen los docentes sobre los métodos y metodología de enseñanza- aprendizaje su clasificación y aplicación en el proceso docente educativo.

La administración del cuestionario se realizó en las aulas de las carreras Agropecuaria y Computación y Redes por los propios investigadores, durante el desarrollo del IX Seminario Científico Metodológico. Antes de entregar el cuestionario, el evaluador proporcionó las instrucciones del llenado y la finalidad de la aplicación del cuestionario. No se limitó el tiempo de respuesta.

El análisis estadístico se realizó a través del uso de la estadística descriptiva, para lo que se determinaron las frecuencias de respuesta para cada pregunta.

Para entender con mejor precisión el dominio de los docentes respecto a los métodos y metodologías de enseñanza- aprendizaje, se analizaron las frecuencias de respuesta a través de un diseño de bloques completamente aleatorizados: Previo al análisis de varianza, se determinó la distribución de normalidad y homogeneidad de varianzas. Las medias de frecuencia se ajustaron a una curva normal, para su respectivo análisis. Tabla \# 2

\section{RESULTADOS Y DISCUSIÓN}

Análisis de la frecuencia en cada carrera

\begin{tabular}{|l|l|l|}
\hline Carreras & Frecuencia & \% \\
\hline Agropecuaria & 8 & 53,3 \\
\hline Computación y Redes & 10 & 50 \\
\hline Total & 18 & 52,4 \\
\hline
\end{tabular}

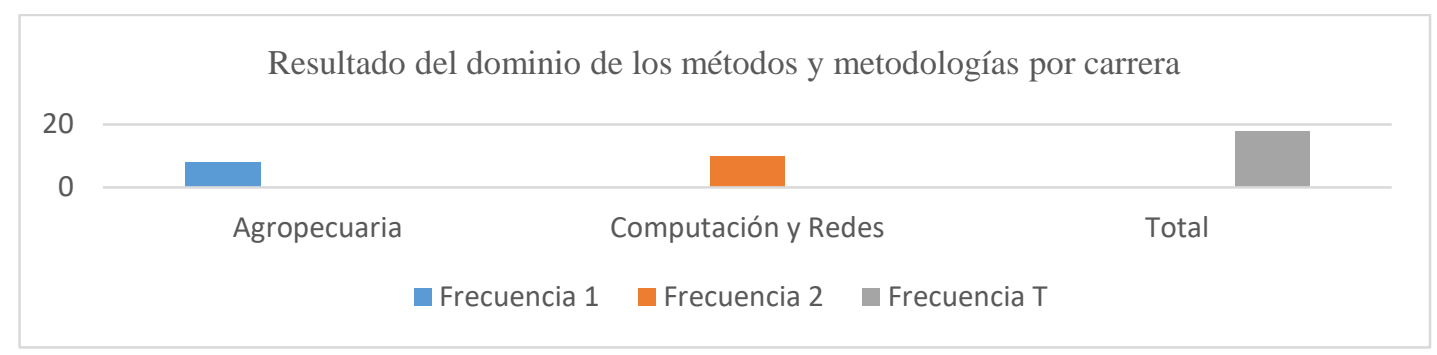

Los resultados de las frecuencias obtenidas en la encuesta nos reflejan que existen dificultades en el dominio, clasificación y utilización de los métodos y metodologías de aprendizaje en las carreras donde se realizó el instrumento.

Las fuentes analizadas arrojaron un total de treinta y ocho métodos de enseñanza. Ahora bien, una de las dificultades consistió en definir cuándo había acuerdo entre los autores respecto a un método concreto. Analicemos que, los autores utilizan términos distintos, y además no siempre definen los métodos en sus listados.

En la tabla \# 3 exponemos una versión revisada del listado, que incorpora treinta y ocho métodos y metodologías de enseñanza aprendizaje con sus definiciones. A pesar de que todas ellas han sido transformadas durante el proceso investigador, por rigor y fidelidad a los autores que las formularon en un inicio hemos resuelto incorporar las definiciones originales teniendo en cuenta 
las fuentes que nombraron los distintos métodos de enseñanza, que es el criterio que hemos seguido para relacionarlos.

\section{MÉTODOS Y METODOLOGÍAS DE ENSEÑANZA-APRENDIZAJE}

\begin{tabular}{|c|c|c|c|}
\hline$\#$ & MÉTODO & DEFINICIÓN ORIGINAL & DEFINICIÓN \\
\hline 1 & $\begin{array}{l}\text { Explicativo- } \\
\text { ilustrativo }\end{array}$ & $\begin{array}{l}\text { Su esencia radica en que el profesor trasmite los } \\
\text { conocimientos, ofrece soluciones a los problemas y hace } \\
\text { demostraciones con la ayuda de diversos medios (exposición, } \\
\text { lectura de libros, demostraciones experimentales, } \\
\text { grabaciones y otros). (Klingberg, Lothar, 1978) }\end{array}$ & $\begin{array}{l}\text { En el método explicativo-ilustrativo, el profesor } \\
\text { transmite conocimientos y el alumno los reproduce; } \\
\text { este método incluye: la descripción, la narración, la } \\
\text { demostración, los ejercicios, la lectura de textos y todo } \\
\text { tipo de recursos para el aprendizaje. }\end{array}$ \\
\hline 2 & $\begin{array}{l}\text { Elaboración } \\
\text { conjunta }\end{array}$ & $\begin{array}{l}\text { La elaboración conjunta por excelencia es la conversación o } \\
\text { el diálogo. El profesor guía la conversación y a los estudiantes } \\
\text { hacia el logro de los objetivos de la clase, los estudiantes } \\
\text { deben participar activamente en la conversación y su } \\
\text { pensamiento se va estimulando por los procesos lógicos del } \\
\text { razonamiento. (Klingberg, Lothar, 1978) }\end{array}$ & $\begin{array}{l}\text { Es el método más utilizado es una conversación o } \\
\text { dialogo, definido como un proceso de } \\
\text { pensamiento colectivo, destacando así la participación } \\
\text { del profesor y los estudiantes en la adquisición de } \\
\text { conocimientos, mediante el papel dirigente del } \\
\text { primero de forma inquisitiva, que exige una respuesta } \\
\text { por parte de los estudiantes. }\end{array}$ \\
\hline 3 & $\begin{array}{l}\text { Trabajo } \\
\text { independiente }\end{array}$ & $\begin{array}{l}\text { Es cuando el estudiante puede relacionar correctamente el } \\
\text { planteamiento de la tarea con los métodos a seguir para } \\
\text { realizarla, cuando puede aplicar sus conocimientos y } \\
\text { capacidades para realizarla sin necesidad de que el maestro } \\
\text { intervenga directamente para orientar cada detalle, es aquel } \\
\text { que se realiza ... (García Batista, Gilberto, 2005) }\end{array}$ & $\begin{array}{l}\text { Consiste en la realización de tareas por parte de los } \\
\text { estudiantes bajo la orientación del profesor. El trabajo } \\
\text { independiente del estudiante debe caracterizarse por el } \\
\text { aspecto productivo que puede alcanzar el nivel más } \\
\text { alto que es la creación. }\end{array}$ \\
\hline 4 & $\begin{array}{l}\text { La conversación } \\
\text { heurística }\end{array}$ & $\begin{array}{l}\text { En el método de conversación heurística se reflejan "...los } \\
\text { resultados del trabajo de búsqueda independiente de los } \\
\text { estudiantes, ya que mediante la discusión se puede orientar la } \\
\text { solución de un problema sobre la base de preguntas y tareas } \\
\text { o de la experiencia personal.(" Martínez Yantada, } \\
\text { M,1987;1999) }\end{array}$ & $\begin{array}{l}\text { En la conversación heurística el profesor establece un } \\
\text { diálogo con el estudiante dirigido mediante preguntas } \\
\text { que van orientando el proceso de razonamiento de los } \\
\text { estudiantes para que arriben a conclusiones similares a } \\
\text { las que llegó el trabajador en la solución del problema } \\
\text { profesional que se manifiesta en el proceso productivo } \\
\text { o de servicios. }\end{array}$ \\
\hline 5 & $\begin{array}{ll}\text { Método } & \text { de } \\
\text { exposición } & \\
\text { problémica } & \end{array}$ & $\begin{array}{l}\text { Mediante este método el docente les enseña a los estudiantes } \\
\text { a hallar la solución a determinado problema revelando } \\
\text { la lógica del mismo a partir de sus contradicciones, indicando } \\
\text { las fuentes de surgimiento del problema, argumentando cada } \\
\text { paso en la búsqueda.(" Martínez Yantada, M, 1998; 85) }\end{array}$ & $\begin{array}{l}\text { El método problémico es donde el profesor } \\
\text { gradualmente va revelando lo fundamental del } \\
\text { conocimiento que imparte, no de forma acabada, } \\
\text { expone problemas y posteriormente en el transcurso de } \\
\text { la clase le va dando solución. }\end{array}$ \\
\hline 6 & $\begin{array}{l}\text { Búsqueda } \\
\text { parcial }\end{array}$ & $\begin{array}{l}\text { La utilización del método de búsqueda parcial "...depende no } \\
\text { sólo del contenido del tema, sino del nivel de la preparación } \\
\text { y capacidad de trabajo de los estudiantes." (Martínez } \\
\text { Yantada, M, 1987; 206) }\end{array}$ & $\begin{array}{l}\text { El este método el profesor, partiendo del problema } \\
\text { docente dado, expone los elementos contradictorios, } \\
\text { no los resuelve, pero estimula la búsqueda } \\
\text { independiente por parte de los alumnos, quienes } \\
\text { analizan documentos, realizan actividades } \\
\text { experimentales y utilizan otras vías para resolver las } \\
\text { contradicciones planteadas por el profesor. }\end{array}$ \\
\hline 7 & Clase magistral. & $\begin{array}{l}\text { Método expositivo, generalmente utilizado para facilitar } \\
\text { información actualizada y bien organizada procedente de } \\
\text { diversas fuentes y de difícil acceso al estudiante (Navaridas } \\
\text { Nalda, F, 2004) }\end{array}$ & $\begin{array}{l}\text { Método cuya finalidad es la exposición de un } \\
\text { contenido actualizado que ha sido elaborado con } \\
\text { finalidad didáctica. }\end{array}$ \\
\hline 8 & Estudio de casos & $\begin{array}{l}\text { Un caso es la descripción real o hipotética que debe ser } \\
\text { estudiada de forma analítica y exhaustiva. Tiene por objetivo } \\
\text { la capacitación práctica para la solución de problemas } \\
\text { concretos (Navaridas Nalda, F, 2004) }\end{array}$ & $\begin{array}{l}\text { Un caso es el relato de una situación que ha sido } \\
\text { articulada con el fin de lograr determinados objetivos } \\
\text { de aprendizaje. El caso debe ser estudiado } \\
\text { exhaustivamente y plantea problemas que los alumnos } \\
\text { deben resolver. }\end{array}$ \\
\hline 9 & Simulación & $\begin{array}{l}\text { Consiste en reproducir acontecimientos o problemas reales } \\
\text { que no están accesibles a los estudiantes y que, sin embargo, } \\
\text { su experimentación la consideramos necesaria para su futuro } \\
\text { profesional (Navaridas Nalda, F, 2004) }\end{array}$ & $\begin{array}{l}\text { Representación de un acontecimiento que } \\
\text { habitualmente no es accesible para el estudiante con el } \\
\text { fin de estudiarlo en un entorno simplificado y } \\
\text { controlado. }\end{array}$ \\
\hline 10 & Proyectos & $\begin{array}{l}\text { Estrategia en la que el producto del proceso de aprendizaje es } \\
\text { un proyecto o programa de intervención profesional, en torno } \\
\text { al cual se articulan todas las actividades formativas } \\
\text { (Fernández March, 2006) }\end{array}$ & $\begin{array}{l}\text { Trabajo cuya finalidad es un producto concreto, } \\
\text { habitualmente condicionada por unos requisitos de } \\
\text { tiempo y recursos, en la que la planificación de tareas }\end{array}$ \\
\hline
\end{tabular}




\section{MÉTODOS Y METODOLOGÍAS UTILIZADOS EN EL PROCESO DE ENSEÑANZA-APRENDIZAJE}

\begin{tabular}{|c|c|c|c|}
\hline & & & $\begin{array}{l}\text { y la resolución de incidencias cobran especial } \\
\text { importancia. }\end{array}$ \\
\hline 11 & $\begin{array}{l}\text { Método } \\
\text { reproductivo }\end{array}$ & $\begin{array}{l}\text { Posibilita el desarrollo de habilidades al proporcionarle al } \\
\text { estudiante un modelo, secuencia de acciones o algoritmo para } \\
\text { resolver una situación con idénticas condiciones. (Klingberg, } \\
\text { Lothar ,1978) }\end{array}$ & $\begin{array}{l}\text { Métodos Reproductivos. Los métodos } \\
\text { reproductivos se caracterizan porque los alumnos } \\
\text { asimilen conocimientos elaborados y reproducen los } \\
\text { modos de actuación que ya conocen. Dentro de este } \\
\text { grupo se incluyen el método explicativo ilustrativo y } \\
\text { el método reproductivo propiamente dicho. }\end{array}$ \\
\hline 12 & Juego de roles & $\begin{array}{l}\text { Un grupo de estudiantes representan una situación de la } \\
\text { realidad, para su posterior análisis por el resto del grupo- } \\
\text { clase. Facilita la comprensión de un problema, vivenciándolo } \\
\text { en la dramatización (Navaridas Nalda, F ,2004) }\end{array}$ & $\begin{array}{l}\text { Representación dramatizada de una situación de la } \\
\text { realidad en que los participantes representan distintos } \\
\text { papeles para su ejercitación y estudio. }\end{array}$ \\
\hline 13 & $\begin{array}{lr}\text { Debate, } & \text { mesa } \\
\text { redonda } & \mathrm{o} \\
\text { coloquio } & \end{array}$ & $\begin{array}{l}\text { Confrontación de opiniones distintas en una discusión } \\
\text { informal, bajo la dirección de un moderador (Navaridas } \\
\text { Nalda, F ,2004) }\end{array}$ & $\begin{array}{l}\text { Confrontación de opiniones en torno a un tema bajo la } \\
\text { dirección de un moderador. Los participantes pueden } \\
\text { alinearse en torno a dos o más posturas dependiendo } \\
\text { del formato. }\end{array}$ \\
\hline 14 & $\begin{array}{l}\text { Aprendizaje } \\
\text { basado en } \\
\text { problemas }\end{array}$ & $\begin{array}{l}\text { Estrategia en la que los estudiantes aprenden en pequeños } \\
\text { grupos partiendo de un problema, a buscar la información que } \\
\text { necesitan para comprender el problema y obtener una } \\
\text { solución, bajo la supervisión de un tutor (Fernández March, } \\
\text { 2006) }\end{array}$ & $\begin{array}{l}\text { Método en el que los estudiantes, en grupo y partiendo } \\
\text { de un problema, determinan sus objetivos de } \\
\text { aprendizaje en función de sus conocimientos y buscan } \\
\text { información para comprender el problema y obtener } \\
\text { una solución con la ayuda de un tutor. }\end{array}$ \\
\hline 15 & $\begin{array}{l}\text { Ejercicios } \\
\text { problemas }\end{array}$ & $\begin{array}{l}\text { Metodología de entrenamiento activa consistente en } \\
\text { identificar una situación conflictiva, definir sus parámetros, } \\
\text { formular y desarrollar hipótesis y proponer una solución o } \\
\text { proponer soluciones alternativas (Navaridas Nalda, F , 2004) }\end{array}$ & $\begin{array}{l}\text { Tarea que consiste en solucionar un problema o } \\
\text { realizar una tarea partiendo de los conocimientos del } \\
\text { estudiante y de una serie de datos que se aportan en el } \\
\text { enunciado del problema. }\end{array}$ \\
\hline 16 & Tutorías & $\begin{array}{l}\text { Se asume la función tutorial como un potente recurso } \\
\text { metodológico que el profesor puede utilizar para } \\
\text { individualizar la enseñanza y ajustarla a las características } \\
\text { personales de cada estudiante (asesorándole sobre la forma de } \\
\text { estudiar la asignatura, facilitándole fuentes bibliográficas y } \\
\text { documentales concretas para la resolución de un problema de } \\
\text { aprendizaje, etc.) (Navaridas Nalda, } F, 2004 \text { ) }\end{array}$ & $\begin{array}{l}\text { Método cuyo fin es individualizar la enseñanza y } \\
\text { ajustarla a las características de cada estudiante } \\
\text { asesorándole sobre la forma de estudiar la asignatura, } \\
\text { facilitándole fuentes bibliográficas, ayudándole en la } \\
\text { resolución de un problema o guiándole en la } \\
\text { realización de un proyecto o investigación. }\end{array}$ \\
\hline 17 & $\begin{array}{l}\text { Prácticas } \\
\text { (laboratorio y } \\
\text { similares) }\end{array}$ & $\begin{array}{l}\text { Modalidad de enseñanza práctica donde el contenido } \\
\text { principal de lo que será aprendido no se proporciona por vía } \\
\text { transmisivo- receptiva, sino que es descubierto por el discente } \\
\text { antes de ser asimilado en su estructura cognitiva (Navaridas } \\
\text { Nalda, F, 2004) }\end{array}$ & $\begin{array}{l}\text { Método de enseñanza práctico y activo donde el } \\
\text { contenido principal de lo que será aprendido es } \\
\text { demostrado o practicado por el alumno, a partir de la } \\
\text { guía del profesor y de unos materiales concreto. }\end{array}$ \\
\hline 18 & $\begin{array}{l}\text { Trabajo } \\
\text { grupo }\end{array}$ & $\begin{array}{l}\text { El profesor programa diversas actividades que deberán } \\
\text { afrontar los equipos de trabajo formados por los estudiantes. } \\
\text { Un buen equipo de trabajo es aquél en que se optimizan las } \\
\text { capacidades de todos los componentes (Navaridas Nalda, F, } \\
\text { 2004) }\end{array}$ & $\begin{array}{l}\text { Realización en equipos de trabajo de actividades } \\
\text { programadas por el profesor que habitualmente } \\
\text { concluye con la elaboración de un documento para su } \\
\text { evaluación. }\end{array}$ \\
\hline 19 & Investigación & $\begin{array}{l}\text { Método de enseñanza práctica que requiere al estudiante } \\
\text { identificar el problema objeto de estudio, formularlo con } \\
\text { precisión, desarrollar los procedimientos pertinentes, } \\
\text { interpretar los resultados y sacar las conclusiones oportunas } \\
\text { del trabajo realizado (Navaridas Nalda, F ,2004) }\end{array}$ & $\begin{array}{l}\text { Método de enseñanza que reproduce las fases y } \\
\text { procedimientos de la investigación científica. Requiere } \\
\text { al estudiante formular el problema, desarrollar las } \\
\text { hipótesis y los procedimientos pertinentes para } \\
\text { contrastarlas, interpretar los resultados y sacar } \\
\text { conclusiones }\end{array}$ \\
\hline 20 & $\begin{array}{l}\text { Estudio } \\
\text { independiente }\end{array}$ & $\begin{array}{l}\text { El trabajo autónomo de los estudiantes permite que cada uno } \\
\text { de ellos vaya siguiendo su propio ritmo y acomodando el } \\
\text { aprendizaje a sus particulares circunstancias (Zabalza, } \\
\text { M.A,2003) }\end{array}$ & $\begin{array}{l}\text { Trabajo autónomo de los estudiantes vinculado a la } \\
\text { materia desarrollada en las clases, que permite que } \\
\text { cada uno siga su propio ritmo acomodando el } \\
\text { aprendizaje a sus particulares circunstancias. }\end{array}$ \\
\hline 21 & $\begin{array}{l}\text { Trabajos o } \\
\text { ensayos } \\
\text { (individuales) }\end{array}$ & $\begin{array}{l}\text { Trabajos que realiza el alumno. Algunos ejemplos pueden } \\
\text { ser: recensiones, trabajos monográficos, memorias o } \\
\text { proyectos (URV, 2006) }\end{array}$ & $\begin{array}{l}\text { Trabajos realizados por los } \\
\text { individualmente, tales costudiantes } \\
\text { monográficos, memorias o ensayos. }\end{array}$ \\
\hline 22 & $\begin{array}{l}\text { Aprendizaje } \\
\text { acción }\end{array}$ & $\begin{array}{l}\text { El aprendizaje acción se construye sobre la relación entre } \\
\text { reflexión y acción. Formaliza el aprendizaje reflexivo y } \\
\text { legitimiza la asignación de tiempo y espacio para ello, a } \\
\text { través de un grupo que trabaja a lo largo de un amplio periodo } \\
\text { de tiempo ( McGill y Brockbank, 2004) }\end{array}$ & $\begin{array}{l}\text { Se lleva a cabo a través de grupos que se reúnen } \\
\text { regularmente donde los integrantes exponen } \\
\text { proyectos, problemas o dificultades que encuentran en } \\
\text { sus entornos de trabajo. Se utiliza el conocimiento y el } \\
\text { apoyo del grupo para elaborar soluciones o propuestas } \\
\text { de mejora. }\end{array}$ \\
\hline
\end{tabular}


Washington Narváez Campana, Cruz Victoria Ponce Zavala, Raquel Vera Velázquez...

\begin{tabular}{|c|c|c|c|}
\hline 23 & $\begin{array}{l}\text { Dinámicas de } \\
\text { grupo }\end{array}$ & $\begin{array}{l}\text { Conjunto de métodos prácticos de trabajo con grupos. } \\
\text { Técnicas (Zapata, Miguel, 2015) }\end{array}$ & $\begin{array}{l}\text { Métodos prácticos y técnicas de trabajo basados en la } \\
\text { dinámica de trabajo basadas en la dinámica grupal. del } \\
\text { grupo. }\end{array}$ \\
\hline 24 & Evaluación & $\begin{array}{l}\text { Pruebas a desarrollar, pruebas de preguntas cortas, pruebas } \\
\text { objetivas tipo test, pruebas prácticas o pruebas orales (URV, } \\
\text { 2006); (Biggs, J, 2004) resume tareas de evaluación y los } \\
\text { tipos de aprendizaje evaluados. }\end{array}$ & $\begin{array}{l}\text { Pruebas de evaluación en diferentes formatos que } \\
\text { pueden incluir preguntas de diverso tipo, pruebas tipo } \\
\text { test, resolución de problemas o pruebas orales. }\end{array}$ \\
\hline 25 & $\begin{array}{l}\text { Prácticas } \\
\text { profesionales }\end{array}$ & $\begin{array}{l}\text { Estadios de formación de la titulación, en general en } \\
\text { empresas o instituciones del sector( URV, 2006) }\end{array}$ & $\begin{array}{l}\text { Segmento de formación en el cual el proceso de } \\
\text { aprendizaje se realiza en empresas o instituciones del } \\
\text { sector, habitualmente bajo la guía de un tutor. }\end{array}$ \\
\hline 26 & Presentaciones & $\begin{array}{l}\text { Exposición oral por parte de los alumnos de un tema concreto } \\
\text { o de un trabajo (previa presentación escrita)( URV, , 2006) }\end{array}$ & $\begin{array}{l}\text { Exposición por parte de los alumnos de un trabajo } \\
\text { previamente desarrollado, normalmente apoyada por } \\
\text { recursos audiovisuales. }\end{array}$ \\
\hline 27 & $\begin{array}{l}\text { Mapas } \\
\text { conceptuales }\end{array}$ & $\begin{array}{l}\text { Consiste en la representación gráfica de los conceptos } \\
\text { fundamentales de la materia de aprendizaje y de sus } \\
\text { relaciones entre sí con el fin de ayudar a los estudiantes a ver } \\
\text { su significado (Navaridas Nalda, F, 2004) }\end{array}$ & $\begin{array}{l}\text { Trabajo cuyo objetivo es la representación gráfica de } \\
\text { los conceptos fundamentales de la materia de } \\
\text { aprendizaje, así como de las relaciones entre ellos. }\end{array}$ \\
\hline 28 & $\begin{array}{l}\text { Método de } \\
\text { dilemas morales }\end{array}$ & $\begin{array}{l}\text { Se trata de una situación problemática, generalmente } \\
\text { presentada de forma oral, a través de la cual los participantes } \\
\text { deberán escoger forzosamente una alternativa, que puede ser } \\
\text { previamente razonada y contrastada en un debate. Su } \\
\text { fundamentación está muy ligada a la clarificación de valores, } \\
\text { ejercicios de toma de decisiones y a los estudios de casos } \\
\text { (Jares, X, 2002) }\end{array}$ & $\begin{array}{l}\text { Se trata de una situación problemática desde el punto } \\
\text { de vista moral en la que los participantes deben escoger } \\
\text { una alternativa que normalmente es previamente } \\
\text { razonada y contrastada en un debate. }\end{array}$ \\
\hline 29 & $\begin{array}{ll}\text { Ejercicio } & \text { de } \\
\text { clarificación } & \text { de } \\
\text { valores } & \end{array}$ & $\begin{array}{l}\text { Resulta útil como vía para tomar consciencia de los códigos } \\
\text { de valoración que tenemos, sensibilizar sobre el sistema de } \\
\text { relaciones en el aula y centro y facilitar su construcción desde } \\
\text { una óptica de respeto y mutua ayuda, así como sobre } \\
\text { diferentes tipos de contenido (procesos de discriminación, } \\
\text { violencia, armamentismo, derechos humanos, desarrollo, } \\
\text { etc.) (Jares, X, 2002) }\end{array}$ & $\begin{array}{l}\text { Tarea que tiene por objeto fundamental tomar } \\
\text { conciencia de los códigos de valoración de cada } \\
\text { participante. }\end{array}$ \\
\hline 30 & Trabajo guiado & $\begin{array}{l}\text { El profesor tutoriza o guía el trabajo de los alumnos a través } \\
\text { de actividades y ejercicios en los que pone en práctica los } \\
\text { contenidos tratados y las competencias. Estas prácticas se } \\
\text { archivan en el portafolio o cuaderno de trabajo y son } \\
\text { evaluadas, de manera que aportan parte de la puntuación total } \\
\text { de la asignatura (20\%), considerando asíl la asistencia a clase.( } \\
\text { ANECA, 2005) }\end{array}$ & $\begin{array}{l}\text { Lo que se aprende depende del contexto, de las } \\
\text { motivaciones de los alumnos, de los descubrimientos } \\
\text { hechos, etc. Es el resultado de las interacciones que la } \\
\text { situación de aprendizaje proporciona. Es decir, es algo } \\
\text { muy poco formalizado pero que integra la complejidad } \\
\text { de forma natural. }\end{array}$ \\
\hline 31 & $\begin{array}{l}\text { Aprendizaje } \\
\text { basado en } \\
\text { proyectos }\end{array}$ & $\begin{array}{l}\text { Método de enseñanza-aprendizaje en el que los estudiantes } \\
\text { llevan a cabo la realización de un proyecto en un tiempo } \\
\text { determinado. Se trata de resolver un problema o abordar una } \\
\text { tarea mediante la planificación, diseño y realización de una } \\
\text { serie de actividades, y todo ello a partir del desarrollo y } \\
\text { aplicación de aprendizajes adquiridos y del uso efectivo de } \\
\text { recursos. (Miguel, M, 2006) }\end{array}$ & $\begin{array}{l}\text { Es un trabajo que se realiza en el aula para crear } \\
\text { servicios o productos únicos. Los estudiantes realizan } \\
\text { una serie de tareas con un tiempo y recursos } \\
\text { determinados, con la continua supervisión y guía del } \\
\text { docente. }\end{array}$ \\
\hline 32 & $\begin{array}{l}\text { Aprendizaje } \\
\text { colaborativo }\end{array}$ & $\begin{array}{l}\text { Una metodología que los maestros usan para agrupar a los } \\
\text { estudiantes e impactar de forma positiva. Quienes utilizan } \\
\text { este método aseguran que hacerlo permite que los estudiantes } \\
\text { mejoren la atención y la adquisición de conocimientos. } \\
\text { (Coll,C, 1990) }\end{array}$ & $\begin{array}{l}\text { El docente debe asegurar una orientación adecuada } \\
\text { para los participantes del hecho colaborativo. } \\
\text { Promover la concientización acerca de lo que se } \\
\text { comparte para colaborar y como se establecen los roles } \\
\text { en la cooperación. Mantener la iniciativa y } \\
\text { construcción propia de conocimientos. }\end{array}$ \\
\hline 33 & $\begin{array}{l}\text { Aprendizaje } \\
\text { conectado }\end{array}$ & $\begin{array}{l}\text { El aprendizaje conectado es un tipo de aprendizaje que integra } \\
\text { el interés personal, las relaciones con los compañeros y los } \\
\text { logros en áreas académicas, cívicos o de la carrera } \\
\text { correspondiente. Además, el aprendizaje conectado es un } \\
\text { enfoque para la reforma educativa centrado en la abundancia } \\
\text { de la información y la conexión social provocada por los } \\
\text { medios de comunicación en red y digitales. (Domínguez } \\
\text { Figaredo, D, 2016) }\end{array}$ & $\begin{array}{l}\text { Guiar hacia las mejores opciones en relación a } \\
\text { esclarecer a los estudiantes, donde los conocimientos } \\
\text { científicos son de mayor calidad y accesibilidad y } \\
\text { Potenciar el uso de las TICS, desde la ecología de } \\
\text { saberes. }\end{array}$ \\
\hline 34 & $\begin{array}{l}\text { Aprendizaje } \\
\text { participativo }\end{array}$ & $\begin{array}{l}\text { Es una metodología que logra que tus clases sean activas y no } \\
\text { exista el aburrimiento. Trata de motivar y conectar con tus } \\
\text { alumnos, aumentando su interés y participación. }\end{array}$ & $\begin{array}{l}\text { Exige asegurar que existan condiciones de } \\
\text { participación. Que la participación } \\
\text { sea real y activa. }\end{array}$ \\
\hline
\end{tabular}

24 UNESUM-Ciencias. Publicación cuatrimestral. Vol. 4, Año 2020, No. 1 (Enero - Abril) 


\begin{tabular}{|c|c|c|c|}
\hline & & $\begin{array}{l}\text { Convirtiéndose el estudiante en el protagonista de su proceso } \\
\text { de aprendizaje.( López Noguero, Fernando, 2005) }\end{array}$ & \\
\hline 35 & $\begin{array}{l}\text { Aprendizaje } \\
\text { interactivo }\end{array}$ & $\begin{array}{l}\text { Conocimiento del medio y sujetos de interacción, se debe } \\
\text { asegurar de la eficacia de los canales para la interactividad } \\
\text { entre los estudiantes y el ambiente, así como el contenido sea } \\
\text { pertinente con los objetivos de las asignaturas y los procesos } \\
\text { pedagógicos. (Díaz Barriga, F,2002) }\end{array}$ & $\begin{array}{l}\text { El Método de Aprendizaje Interactivo de convierte al } \\
\text { estudiante en el centro del proceso educativo } \\
\text { adaptándose a sus horarios, ritmo y métodos de } \\
\text { aprendizaje. }\end{array}$ \\
\hline 36 & $\begin{array}{l}\text { Aprendizaje } \\
\text { contextualizado }\end{array}$ & $\begin{array}{l}\text { Independientemente de cómo se lleva a cabo, dentro de la } \\
\text { multiplicidad en la literatura, la contextualización curricular } \\
\text { es una forma de trabajo pedagógico que se pretende } \\
\text { relacionar los contenidos de enseñanza-aprendizaje y el } \\
\text { currículo a las realidades sociales y culturales de los } \\
\text { estudiantes, sus conocimientos. (Álvarez, R. P ,2004) }\end{array}$ & $\begin{array}{l}\text { Es un estudio previo de los escenarios y los contextos. } \\
\text { Que los aprendizajes tengan una relación entre } \\
\text { tensiones y problemas conocidos. }\end{array}$ \\
\hline 37 & $\begin{array}{l}\text { El aprendizaje } \\
\text { invertido }\end{array}$ & $\begin{array}{l}\text { El aprendizaje invertido (conocido por el nombre de flipped } \\
\text { learning, en inglés) es un método de aprendizaje semi } \\
\text { presencial que consiste en dictar las lecciones fuera del salón } \\
\text { de clase, por ejemplo, a través de la modalidad a distancia, y } \\
\text { utilizar las clases presenciales para practicar y aplicar esos } \\
\text { nuevos conceptos, a través de actividades prácticas o debates. } \\
\text { (Díaz Barriga, F ,2002) }\end{array}$ & $\begin{array}{l}\text { El cometido de esta propuesta es esencialmente } \\
\text { asegurarse de que los alumnos hayan comprendido } \\
\text { verdaderamente lo que se les ha enseñado, sacar el } \\
\text { máximo provecho del tiempo limitado que estos } \\
\text { comparten junto a los profesores, y, sobre todo, } \\
\text { involucrarlos activamente en el proceso de aprendizaje } \\
\text { y favorecer el pensamiento crítico. }\end{array}$ \\
\hline 38 & $\begin{array}{l}\text { Aprendizaje } \\
\text { activo }\end{array}$ & $\begin{array}{l}\text { Esta es una estrategia que busca el compromiso del } \\
\text { estudiante con su estudio y el aprendizaje a través de } \\
\text { la colaboración con otros compañeros, la reflexión } \\
\text { individual, el pensamiento crítico y la acción directa para } \\
\text { resolver problemas. (Álvarez, R. P, 2004) }\end{array}$ & $\begin{array}{l}\text { Los expertos indican que esta estrategia implica } \\
\text { una inversión de la clase, lo que se señala debido a que } \\
\text { en el aprendizaje activo el estudiante deja de ser } \\
\text { considerado como un elemento a moldear o llenar de } \\
\text { información, y pasa a tener un rol activo en su } \\
\text { aprendizaje. Es decir, se aleja del modelo tradicional } \\
\text { de escuchar al docente de forma pasiva y se acerca a } \\
\text { la toma de decisiones y búsqueda de información de } \\
\text { manera individual, pero orientada por el docente. }\end{array}$ \\
\hline
\end{tabular}

Nuestra investigación se basó en las fuentes que hemos mencionado en el desarrollo de la investigación, basado en un número abundante de autores. Se trata de autores muy diversos: algunos son reflexiones sobre la docencia universitaria, otros son trabajos de investigación y los demás hablan acerca de la experiencia en la educación en general. Decidimos incluirlos todos basándonos en el análisis de (Álvarez de Zayas, 1999), en el que se recoge que el nivel académico de los estudiantes no influye de modo significativo en el efecto de un determinado método. Por tanto, a priori lo que funciona en un nivel educativo puede funcionar igualmente en otro de cada uno de ellos partimos del cuestionario de métodos de enseñanza que conocemos que incorpora un glosario, que es el de Navaridas Nalda, F (2004). En el caso de que la definición que aportaba el glosario a nuestro entender pudiera ser mejorada intentamos hacerlo, y en el caso de métodos no recogidos por el cuestionario recurrimos a fuentes externas.

Así se construyó la versión del listado, inicialmente elaborado en el marco de una investigación sobre la estrategia docente de las instituciones de Educación Superior, (Alcoba, 2012). Este listado sirvió en dicho estudio para lograr los siguientes objetivos:

- Utilizar un lenguaje común a la hora de referirse a los métodos de enseñanza.

- Servir de base para la elaboración de un cuestionario a través del cual se analizó el género metodológico de la institución en la cual tuvo lugar el estudio.

- Guiar los procesos de formación de los docentes.

- Elaborar los sílabos de las diferentes materias. 
Washington Narváez Campana, Cruz Victoria Ponce Zavala, Raquel Vera Velázquez...

La ausencia de terminología unificada en el campo de los métodos de enseñanza es un hecho constatado, y por eso es común en este terreno que los autores comiencen por definir el significado que para ellos tienen los términos que usan (Fernández Pérez, 1994, p. 584)

En este trabajo utilizamos la expresión método de enseñanza-aprendizaje para referirnos a la unidad básica de la metodología. En la revisión de listados de métodos de enseñanza que realizamos en este trabajo, quince autores utilizaron este término (Hernández, P ,1997); (Bourner, T ,1997); (Labarrere, G y Valdivia P, G.E, 1991); (Silvestre Oramas, M, 2002); (García Batista, G ,2002); (Álvarez, R. P ,2004); (Miguel, M, 2006); (Fernández, March ,2006); (Miguel, M (coord.), 2006); (BusinessWeek, 2008); (Franco, César ,2010); (Caballero, C.E ,2012) y (Zapata, Miguel, 2015)

Los otros términos utilizados fueron: métodos y métodos didácticos Euro, (F.M, 2007); (Zabalza, M.A, 2003), técnicas docentes (Fernández Serrat, M.L, 2007), metodologías educativas (MEC ,2006); (BIGGS, J, 2004); (Addine Fernández, F ,2004) estrategias de enseñanzaaprendizaje (Andreu, M. A. et. al , 2005) estrategias metodológicas (Gairín, J ,2005) métodos formativos López Camps, J (2005) estrategias didácticas Navaridas Nalda, F (2004), métodos pedagógicos Amat, O (2002) actividades didácticas (Jares, X , 2002), y metodologías docentes (Fernández Glaser, A y Sarramona, J ,1975); (Hernández, P ,1997); (Universitat Rovira I Virgili ,2006) y desde el punto de vista del constructivismo, se considera que la metodología debe reunir varias características, que ya son mencionadas en otras fuentes y de las cuales se hace un resumen a continuación (Universidad San Buenaventura y Zapata, Miguel ,2015)

Ddesde nuestra perspectiva un método de enseñanza es el conjunto de técnicas y actividades que un profesor utiliza con el fin de lograr uno o varios objetivos educativos, que tiene sentido como un todo y que responde a una denominación conocida y compartida por la comunidad científica.

Resaltamos que método es un término de índole más genérica que actividad o técnica, pero más concreta que modelo, y destacamos la relación que existe entre el método y los fines que pretende, que bajo nuestro punto de vista no son otros que las finalidades educativas en sus diversos niveles de concreción. Ahora bien, para que un método sea tal (y no una técnica o una actividad), debe tener sentido como un todo, es decir, debe poder diferenciarse claramente cuándo un profesor está usando un método y cuándo otro. Así, en su ejecución, la clase magistral no debe ser lo mismo que el método del caso, ni la resolución de ejercicios y problemas deben ser lo mismo que las prácticas profesionales. Por último, un método de enseñanza debe tener un nombre reconocido y compartido por la comunidad científica. Un método no lo es hasta que se investiga y comunica sobre él. Un profesor puede diseñar un gran número de actividades, pero los métodos (y probablemente las técnicas) pertenecen a una escala de complejidad superior y para constituirse como tales debe haber cierto acuerdo en la comunidad científica sobre ellos.

Para nosotros por tanto la metodología es la relación de métodos de enseñanza que usa un profesor y su articulación en el aula y no, por ejemplo, la forma que este tiene de adjetivarlos. En otras palabras, lo que un profesor ha de consignar en el apartado de metodología dentro de una guía académica, como mínimo, es una enumeración de los métodos que usa y no, o al menos no solo, si esta es activa, si es participativa o si sigue o no los principios del aprendizaje significativo.

\section{CONCLUSIONES}

Se consultó una serie de autores para realizar el análisis de los puntos de vista de cada autor y se construyó un listado de treinta y ocho métodos y metodologías de enseñanza-aprendizaje, para 
tener un material didáctico donde puedan nuestros docentes consultar para desarrollar su proceso de enseñanza y aprendizaje con mayor calidad.

Este estudio sirvió para lograr los objetivos propuestos como utilizar un lenguaje común al referirse a los métodos y metodologías de enseñanza-aprendizaje, servir de base para tener un material didáctico para las carreras e institución que fueron objetos de estudio, guiar los procesos de preparación de los docentes y elaborar los sílabos de las diferentes asignaturas.

El modelo planteado trae como objetivo la investigación docente y pone de manifiesto que la ciencia de los métodos y metodologías está más actual que nunca en nuestro proceso de enseñanzaaprendizaje.

\section{REFERÉNCIAS BIBLIOGRÁFICAS}

Addine Fernández, F. (2004). Didáctica: teoría y práctica. La Habana: Pueblo y Educación.

Alcoba González, J. (2012). La clasificación de los métodos de enseñanza en educación superior. Contextos Educativos, 15. Madrid.

Álvarez de Zayas, C. (1999). Didáctica: la escuela en la vida. La Habana: Pueblo y Educación.

Álvarez, R. P. (2004). Formación superior basada en competencias, interdisciplinariedad y trabajo autónomo del estudiante. Revista Iberoamericana de Educación. Disponible en http://www. rieoei.

Amat, O. (2002). Aprender a enseñar. Una visión práctica de la formación de formadores. Barcelona. España.

Andreu, M. A. et. al. (2005). Estrategias de enseñanza-aprendizaje en la Universidad Politécnica de Valencia. Disponibleen:http://www.upct.es/ dcta/Convergencia/Otros_documentos/Estrategias_de_ensenanza_en_la_UPV.pdf

ANECA (2005) Libro Blanco. Título de grado en Magisterio. Volumen 1. Madrid: Agencia Nacional de Evaluación de la Calidad y Acreditación.

Biggs, J. (2004). Calidad en el aprendizaje universitario. Madrid: Nancea.

Bourner, T. (1997). Teaching methods for learning outcomes. Education + Training, 39(9), 344-348.

Businessweek. (2008). Full-Time MBA Profiles. Disponible http://www.businessweek.com/bschools/06/geographic.htm.

Caballero C, E. (2012). Didáctica de la escuela primaria. Selección de lecturas. La Habana: Pueblo y Educación.

Díaz Barriga F, Hernández G. (2002). Estrategias docentes para un aprendizaje significativo. Una interpretación constructivista. México, DF: McGraw-Hill I

Domínguez Figaredo, D. (2016). Aprendizaje conectado: LRNG. Master en Educación y Comunicación La Red, Asignatura Gestión del conocimiento en la red

Fernández March, A. (2006). Metodologías activas para la formación de competencias. Educativo Siglo XXI, 24, 35 56.

Fernando López Noguero Madrid, Narcea, (2005 175 pp) "Metodología Participativa En La Enseñanza Universitaria" Revista Interuniversitaria de Formación del Profesorado, vol. 20, núm. 3, 2006, pp. 313-316 Universidad de Zaragoza, España

Fernández, A. y Sarramona, J. (1975). La Educación: constantes y problemática actual. Barcelona: CEAC.

Fernández Serrat, M.L. (2007). Planificación del Aprendizaje. En M.C. Fonseca Mora y J. I. Aguaded Gómez (Dir), Enseñar en la Universidad. Experiencias y Propuestas para la Docencia Universitaria.

Gairín, J. (2005). Nociones de desarrollo curricular. El crédito europeo. En C. Chamorro y P. Sánchez (Eds.), Iniciación a la docencia universitaria. Manual de ayuda. Madrid: Universidad Complutense de Madrid-ICE.

García Batista, G. (2002). Compendio de Pedagogía. La Habana: Pueblo y Educación. 
Washington Narváez Campana, Cruz Victoria Ponce Zavala, Raquel Vera Velázquez...

García Batista, Gilberto. "El trabajo independiente. Sus formas de realización.”, Editorial Pueblo y Educación, Cuba, 2005.007

Hernández, P. (1997). Construyendo el constructivismo: criterios para su fundamentación y su aplicación instruccional. En M. J. Rodrigo, y J. Arnay, (Comp.): La construcción del conocimiento escolar. Barcelona: Paidós.

Jares, X. (2002). Métodos y actividades didácticas. En M. Rodríguez Rojo (Ed.), Didáctica General. Qué y cómo enseñar en la sociedad de la información. Madrid: Biblioteca Nueva.

Jiménez, H. y Hernández-Jorge, C. (2001). “CCómo motivar a los alumnos para que aprendan?: la motivación en la enseñanza".

Klingberg, Lothar. Introducción a la Didáctica General. La Habana, Pueblo y Educación. 1978.

Klingberg, L. (1972). Introducción a la Didáctica General. La Habana: Pueblo y Educación.

Labarrere, G. y Valdivia P., G.E. (1991). Pedagogía. La Habana: Pueblo y Educación.

López Camps, J. (2005). Planificar la formación con calidad. Madrid: CissPraxis.

Martínez Llantada, Martha .(1987): La enseñanza problémica de la Filosofía Marxista Leninista. Editorial de Ciencias Sociales. La Habana.

Martínez Llantada, Martha. (1998). Calidad educacional, actividad pedagógica y creatividad. Editorial Academia. La Habana.

McGill, I y Brockbank, A. (2004). The action learning handbook. Londres: RoutledgeFalmer.

MEC. (2006). Propuestas para la renovación de las metodologías educativas en la universidad. Madrid: Secretaría de Estado de Universidades e Investigación. Dirección General de Universidades. MEC.

Miguel, M. (coord.) (2006). Metodología de enseñanza y aprendizaje para el desarrollo de competencias. Orientaciones para el profesorado universitario ante el EEES. Madrid: Alianza.

Navaridas Nalda, F. (2004). Estrategias didácticas en el aula universitaria. Logroño: Universidad de la Rioja. Servicio de Publicaciones.

Ortega Loubon, C. y J. César Franco. (2010). Neurofisiología del aprendizaje y la memoria. Plasticidad Neuronal.Archivos de Medicina, 6 (1), 1-2, Panamá. 